\title{
Erratum
}

\section{Further Establishment of the Field Perturbation Theory of the Pseudogaps in HTSC}

\section{Moshe Dayan ${ }^{1}$}

Erratum to: Journal of Superconductivity and Novel Magnetism, Vol. 20, No. 3, p. 239 (2007)

DOI: $10.1007 / \mathrm{s} 10948-006-0184-5$

1. On page 242 (top left column) the equal signs are missing from the equations. The correct equations are as follows:

$$
M_{k}=\left[\begin{array}{ll}
u_{k}^{2} & -u_{k} v_{k} \\
-u_{k} v_{k} & v_{k}^{2}
\end{array}\right],
$$

and

$$
N_{k}=\left[\begin{array}{ll}
v_{k}^{2} & u_{k} v_{k} \\
u_{k} v_{k} & u_{k}^{2}
\end{array}\right] .
$$

2. There is an error in equation 17 . The correct equation is as follows:

$$
\begin{aligned}
H_{0}(x)= & \frac{1}{2} \sum_{k, s}\left\{\varepsilon_{k} \tilde{\Psi}_{k, s}^{\dagger} \tau_{3} \tilde{\Psi}_{k, s}+\Lambda_{k}\left[\tilde{\Psi}_{k, s}^{\dagger} \tilde{\Psi}_{k, s}\right.\right. \\
& \left.\left.+\tilde{\Psi}_{k, s}^{\dagger}(x) \tau_{1} \tilde{\Psi}_{k, s}(x)\right]+\left(E_{k}-\Lambda_{k}\right)\right\} .
\end{aligned}
$$

3. On page 244 there is an error in the first sentence of the last paragraph. The correct sentence is as follows:

Thus, we may have momentum-defined interactions that are terminated by two $I$ vertices, and momentum-defined interactions that are terminated by two $\tau_{1}$ vertices.

4. The page number is incorrect in Ref. 6. The correct reference is as follows:

M. Dayan, J. Supercon. 19, 477 (2006).
The online version of the original article can be found at: http://dx.doi.org/10.1007/s10948-006-0184-5

\footnotetext{
${ }^{1}$ Department of Physics, Ben-Gurion University, Beer-Sheva 84105, Israel; e-mail: mdayan@bgu.ac.il
} 